\title{
Uso popular de espécies medicinais da família Verbenaceae no Brasil
}

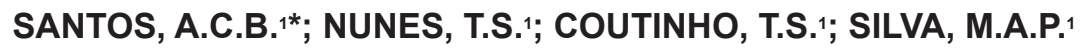

Universidade Regional do Cariri - URCA, Programa de Pós-graduação em Bioprospecção Molecular, Rua Cel. Antônio Luis, 1161, Pimenta, Crato, CE, CEP: 63100-000. *Autor para correspondência: carlito.santos@urca.br

\begin{abstract}
RESUMO: Com o presente trabalho objetivou-se realizar um levantamento bibliográfico acerca das espécies da família Verbenaceae utilizadas na medicina popular nas diversas regiões do Brasil. Os trabalhos acadêmicos utilizados para elaboração desta pesquisa foram extraídos de revistas indexadas junto ao Scielo publicadas no período de 2000 a 2012, sendo também realizada busca no Google acadêmico. Os dados foram compilados e organizados em ordem alfabética por espécies, seguidas do nome popular, forma de uso, parte usada, indicações e área de estudo. Foram encontrados um total de 85 publicações indicando 55 espécies distribuídas em oito gêneros. A espécie com maior número de citações foi Lippia alba (Mill.) N.E.Br., com 56 registros. Das plantas descritas na literatura, a folha foi o órgão mais citado como parte usada e o chá, o modo de preparo mais utilizado. Dentre as categorias de indicações medicinais, as plantas que mereceram destaque foram às relacionadas ao tratamento das doenças do sistema respiratório e digestório. Constatou-se que o Rio de Janeiro foi o estado que contou com o maior número de publicações na área. O uso de plantas medicinais está cada vez mais presente entre as diversas comunidades. Com isso, as informações contidas neste trabalho servirão para auxiliar na elaboração de banco de dados sobre o modo de uso das plantas pertencentes à família Verbenaceae com potencial medicinal encontradas nos Estados brasileiros.
\end{abstract}

Palavras-chave: Recursos Vegetais, Comunidade tradicional, Plantas medicinais.

\begin{abstract}
Popular use of medicinal species of the Verbenaceae family in Brazil. The current work aimed to conduct a literature review about the Verbenaceae family species used in popular medicine in different regions of Brazil. The academic papers employed for the preparation of this research were taken from journals indexed by the Scielo published from 2000 to 2012, and a research in Google scholar tool was also performed. The data were compiled and organized alphabetically by species, followed by the popular name, manner of use, employed part, indications and study area. A total of 85 publications indicating 55 species were found and distributed into eight genera. The species with the highest number of citations was the Lippia alba (Mill.) N.E.Br., with 56 records. From the plants described in the literature, the leaf was the most cited part of the vegetables, and the tea was the most widely used preparation method. Among the categories of medicinal recommendations, the highlighted plants were the ones related to the treatment of diseases of the respiratory and the digestive systems. It was observed that Rio de Janeiro was the state in which the largest number of publications in the area was concentrated. The use of medicinal plants is increasingly present among several communities. Thus, the information in this work will be applied to assist in database development on how to use the plants belonging to the family Verbenaceae with medicinal potential found in Brazilian states.
\end{abstract}

Keywords: Plant Resources, Traditional community, Medicinal plants.

\section{INTRODUÇÃO}

Desde épocas remotas, as sociedades acumulam informações e experiências sobre o ambiente em que vivem, para com ele interagir e prover suas necessidades de sobrevivência. Uma interação importante vem das plantas que ao longo do desenvolvimento das civilizações apresentaram importantes utilidades, servindo não só como fonte de alimento e uso ornamental, mas também como 
forma de obtenção de medicamentos, cosméticos e outros (Badke et al., 2012). Essas interações entre seres humanos e plantas são moldadas pela história, pela cultura, pelos ambientes físico, biológico, social e pelas características inerentes às plantas (Araújo, 2007).

O uso medicamentoso das plantas é muito frequente entre a população mais pobre, sendo cultivadas e até comercializadas, o que contribui com a divulgação do seu efeito de acordo com o uso popular, despertando o interesse de estudos sobre uma determinada espécie, aliando o conhecimento popular a medicina (Maciel et al., 2002).

O conhecimento de plantas medicinais representa em muitas vezes o único recurso terapêutico de comunidades e grupos éticos, sendo que a procura por tratamentos alternativos ou aliados a medicamentos, vem aumentando consideravelmente, tornando essa prática frequente entre a sociedade brasileira (Silva \& Hahn, 2011).

A etnobotânica tem sido objeto de estudo no mundo e no Brasil, onde as diversas áreas de investigação tentam resgatar o conhecimento popular a respeito dos vegetais, seus usos e especialmente ao uso medicinal. A intensificação dos trabalhos etnobotânicos leva a conhecer essas espécies e poderá servir como instrumento para traçar estratégias de utilização das espécies nativas e seus potenciais (Ming et al., 2000).

A família Verbenaceae compreende cerca de 98 gêneros e 2614 espécies no mundo. No Brasil, encontram-se 47 gêneros distribuídos em 407 espécies nos diferentes hábitos, desde ervas perenes, arbustos até subarbustos, encontrados nas regiões tropicais e subtropicais (Salimena et al., 2013; Vandresen, 2005), apresentando distribuição pantropical, mas principalmente neotropical (Bueno \& Leonhardt, 2011). As espécies estão distribuídas em todas as regiões do país, ocorrendo em vegetações do tipo Campo Rupestre, Cerrado (latu sensu) e Floresta Ombrófila (Floresta Pluvial), sendo presente em diversos domínios fitogeográfico, como: Amazônia, Caatinga, Cerrado, Mata Atlântica e Pampa (Salimena et al., 2013). Suas espécies apresentam diferentes aplicações, podendo ser utilizadas pelas propriedades medicinais de algumas espécies, bem como ornamentais e madeiras, sendo esta última amplamente empregada na economia (Melo et al., 2010). Uma característica da família é a presença de tricomas secretores, geralmente produtores de óleos essenciais de grande valor medicinal (Favorito, 2009).

Diante dessas considerações, no presente trabalho objetivou-se realizar um levantamento bibliográfico de artigos publicados no período de 2000-2012 acerca das espécies de Verbenaceae utilizadas na medicina popular nas diversas regiões do Brasil, contribuindo dessa forma, para a conservação destas informações, assim como, dando subsídio para posteriores investigações no âmbito farmacológico científico no intuito de comprovar as propriedades referidas nesta pesquisa.

\section{MATERIAL E MÉTODOS Coleta de dados}

O estudo foi baseado em um levantamento bibliográfico no período de 2000 a 2012 sobre as espécies de Verbenaceae com potencial medicinal utilizada por comunidades brasileiras.

Os trabalhos acadêmicos utilizados para elaboração desta pesquisa foram extraídos de revistas indexadas junto ao Scielo, sendo também realizada busca no google acadêmico. As palavraschave utilizadas como fonte de pesquisa foram: Verbenaceae, Etnobotânica, Plantas Medicinais, Uso de Recursos Vegetais e Medicina popular.

Para revisão dos nomes científicos e sinonímias das espécies citadas foram utilizados os portais da Lista de espécies da Flora do Brasil (Koch et al., 2014) e Tropicos (2014), baseado na classificação do APG III (2009).

\section{Registro das Espécies da Família Verbenaceae}

No levantamento etnobotânico da família Verbenaceae, foram registrados um total de 85 publicações indicando 55 espécies distribuídas em oito gêneros, sendo que 36 foram identificadas a nível de espécie, 19 somente a nível de gênero e quatro não identificadas. Das espécies identificadas apenas a nível de gênero, Lippia sp. foi a mais representativa com 12 indicações (Tabela 1).

As espécies com maior número de citações foram Lippia alba (Mill.) N.E.Br., Stachytarpheta cayannensis (Rich.) Vahl. e Lantana camara L. com 56,35 e 25 citações, respectivamente. Já dentre os gêneros listados, aqueles com maior números de espécies foram Lippia spp. (22 registros), seguidos de Lantana spp. (9), Stachytarpheta spp. (7), Aloysia spp. (6) e Verbena spp. (4).

O trabalho de Brito \& Senna-Valle (2011) desenvolvido em Paraty (RJ) mostrou que Lippia alba (Mill.) N. E. Br. (erva-cidreira) apresentou o maior valor de Importância Relativa, ou seja, foi a espécie indicada para o tratamento de um maior número de sintomas.

Com relação à atividade farmacológica de Lippia alba (Mill.) N.E.Br, essa espécie apresenta várias ações, sendo o calmante a mais utilizada (Aguiar \& Barros, 2012; Borba \& Macedo, 2006). Camêlo et al. (2011) e Favorito (2009) afirmaram ainda, que $L$. alba possui características que

Rev. Bras. PI. Med., Campinas, v.17, n.4, supl. II, p.980-991, 2015. 
TABELA 1. Espécies medicinais da família Verbenaceae, seguidas pelo Nome Popular, Forma de Uso, Parte Usada, Indicações, Área de Estudo e Referências.

\begin{tabular}{|c|c|c|c|c|c|c|}
\hline Nome Científico & Nome Popular & $\begin{array}{l}\text { Forma de } \\
\text { Uso }\end{array}$ & $\begin{array}{l}\text { Parte } \\
\text { Usada }\end{array}$ & Indicações & Área de Estudo & Referências \\
\hline $\begin{array}{l}\text { Aloysia citriodora } \\
\text { Palau } \\
\text { [Sin.Aloysia } \\
\text { triphylla (L'Hér.) } \\
\text { Britton] }\end{array}$ & $\begin{array}{l}\text { Cidró, cidró- } \\
\text { pessegueiro, } \\
\text { cidrão, cidreira, } \\
\text { cidrozinho, erva- } \\
\text { Luíza, ervacidreira }\end{array}$ & Chá & Fo & $\begin{array}{l}\text { Adstringente, sedativo } \\
\text { brando, febre, sistema } \\
\text { digestivo, inseticida, } \\
\text { bactericida, resfriado, } \\
\text { tônica, antiespasmódica, } \\
\text { carminativa, eupéptica, } \\
\text { calmante, anti-gripal, } \\
\text { coração, estimulante, } \\
\text { probema nervoso, acne }\end{array}$ & $\begin{array}{l}\text { Anita Garibaldi (SC) } \\
\text { Cascavel (PR) } \\
\text { Ipê (RS) } \\
\text { Itapoá (SC) } \\
\text { Lages (SC) }\end{array}$ & $\begin{array}{l}\text { Lorenzi \& Matos (2008) } \\
\text { Maia et al. (2011) } \\
\text { Merétika et al. (2010) } \\
\text { Negrelle et al. (2007) } \\
\text { Ritter et al. (2002) }\end{array}$ \\
\hline $\begin{array}{l}\text { A. gratissima } \\
\text { (Gillies \& Hook. } \\
\text { ex Hook.) Tronc. }\end{array}$ & $\begin{array}{l}\text { Alfazema, } \\
\text { alfazema-do- } \\
\text { Brasil, alfazema- } \\
\text { macho,novalgina- } \\
\text { em-folhas }\end{array}$ & Banho & $\begin{array}{l}\mathrm{Fo}, \mathrm{Cl} \\
\mathrm{Pt}\end{array}$ & $\begin{array}{l}\text { Dor no corpo, febre, } \\
\text { dor de cabeça, } \\
\text { doenças da pele, } \\
\text { infecções brônquicas, } \\
\text { afecções pulmonares, } \\
\text { antimicrobiana }\end{array}$ & $\begin{array}{l}\text { Barra do Piraí (RJ) } \\
\text { Casimiro de Abreu } \\
\text { (RJ) } \\
\text { Paraty (RJ) } \\
\text { Quissamã (RJ) } \\
\text { Santa Leopodina } \\
\text { (ES) }\end{array}$ & $\begin{array}{l}\text { Borges \& Peixoto (2009) } \\
\text { Boscolo \& Valle (2008) } \\
\text { Christo et al. (2010) } \\
\text { Crepaldi (2007) } \\
\text { Parente \& Rosa (2001) } \\
\text { Santos et al. (2009) }\end{array}$ \\
\hline $\begin{array}{l}\text { A. IycioidesCham. } \\
\text { [Sin.A. pulchra } \\
\text { (Briq.) Moldenke] }\end{array}$ & Cidró & Chá & Fo & Antigripal & Cascavel (PR) & Negrelle et al. (2007) \\
\hline $\begin{array}{l}\text { A. oblanceolata } \\
\text { Moldenke }\end{array}$ & $\begin{array}{l}\text { Camomila, } \\
\text { pau-de-angola- } \\
\text { pequeno }\end{array}$ & & & & $\begin{array}{l}\text { Macapá (AP) } \\
\text { Ouro Verde de } \\
\text { Góias }(G O)\end{array}$ & $\begin{array}{l}\text { Silva (2002) } \\
\text { Silva \& Proença (2008) }\end{array}$ \\
\hline Aloysia sp1. & $\begin{array}{l}\text { Alecrim-do-chile, } \\
\text { alcanflor }\end{array}$ & & & & Anastácio (MS) & $\begin{array}{l}\text { Cunha \& Bortolotto } \\
(2011)\end{array}$ \\
\hline \multirow[t]{2}{*}{ Aloysia sp2. } & Erva-santa & & & $\begin{array}{l}\text { Intestino preso, dor } \\
\text { no estômago, dor de } \\
\text { barriga, diarréia, gastrite, }\end{array}$ & Florianópolis (SC) & $\begin{array}{l}\text { Giraldi \& Hanazaki } \\
(2010)\end{array}$ \\
\hline & & & & $\begin{array}{l}\text { enjôo, induzir vômito, } \\
\text { gases, má digestão, } \\
\text { congestão, queimor no } \\
\text { estômago, purgante, } \\
\text { laxante. }\end{array}$ & & \\
\hline $\begin{array}{l}\text { Bouchea } \\
\text { fluminensis (Vell.) } \\
\text { Moldenke } \\
\text { [Sin.B. } \\
\text { pseudogervao } \\
\text { (A.St.-Hil.) } \\
\text { Cham.] }\end{array}$ & $\begin{array}{l}\text { Gervão-de-folha- } \\
\text { grande }\end{array}$ & & Fo & Úlceras & & Fenner et al. (2006) \\
\hline Bouchea sp. & Gervão-roxo & & & & $\begin{array}{l}\text { Nova Friburgo (RJ) } \\
\text { Petrópolis (RJ) }\end{array}$ & Leitão et al. (2009) \\
\hline $\begin{array}{l}\text { Casselia } \\
\text { chamaedryfolia } \\
\text { Cham. } \\
\text { [Sin. Casslia } \\
\text { mansoi Schauer] }\end{array}$ & Saúde-da-mulher & & & & Mato Grosso & $\begin{array}{l}\text { Guarim Neto \& Morais } \\
(2003)\end{array}$ \\
\hline $\begin{array}{l}\text { Lantana } \\
\text { caatingensis } \\
\text { Moldenke }\end{array}$ & & & & & Alagoinha (PE) & $\begin{array}{l}\text { Albuquerque et al. } \\
(2005)\end{array}$ \\
\hline L. camara L. & $\begin{array}{l}\text { Bem-me-quer, } \\
\text { cambará, } \\
\text { cambará-de- } \\
\text { chumbo, cambará- } \\
\text { de-espinho, } \\
\text { cambará-de- } \\
\text { cheiro, }\end{array}$ & $\begin{array}{l}\text { Infusão, } \\
\text { decocto, } \\
\text { extrato } \\
\text { fluido, } \\
\text { xarope, } \\
\text { elixir, banho }\end{array}$ & $\begin{array}{l}\mathrm{Fo}, \mathrm{FI} \\
\mathrm{Sm}\end{array}$ & $\begin{array}{l}\text { Afecção pulmonar, asma, } \\
\text { bronquite, dor de ouvido, } \\
\text { espasmo, febre, peitoral, } \\
\text { reumatismo, tosse } \\
\text { catarral, coqueluche, } \\
\text { vias respiratórias, enjoo, } \\
\text { catapora, doenças no } \\
\text { fígado, higiene íntima, }\end{array}$ & $\begin{array}{l}\text { Alagionha (PE) } \\
\text { Amargosa (BA) } \\
\text { Anastacio (MS) } \\
\text { Belo Horizonte } \\
\text { (MG) } \\
\text { Boa Vista (RR) } \\
\text { Santa Cruz Cabrália } \\
\text { (BA) } \\
\text { Carrancas (MG) }\end{array}$ & $\begin{array}{l}\text { Agra et al. (2008) } \\
\text { Alves et al. (2000) } \\
\text { Amorozo (2002) } \\
\text { Boscolo \& Valle (2008) } \\
\text { Botrel et al. (2006) } \\
\text { Brandão et al. (2006) } \\
\text { Brandão et al. (2008) }\end{array}$ \\
\hline
\end{tabular}


TABELA 1. Espécies medicinais da família Verbenaceae, seguidas pelo Nome Popular, Forma de Uso, Parte Usada, Indicações, Área de Estudo e Referências.

continuação...

\begin{tabular}{|c|c|c|c|c|c|c|}
\hline & $\begin{array}{l}\text { cambará-de- } \\
\text { folha-grande, } \\
\text { cambará-miúdo, } \\
\text { cambarazinho, } \\
\text { camarazinho, } \\
\text { cambará- } \\
\text { vermelho, } \\
\text { capitão-de-campo, } \\
\text { milho-de-grilo, } \\
\text { carará-juba, } \\
\text { cariaquito- } \\
\text { vermelho, } \\
\text { chumbinho, } \\
\text { erva-chumbinho, } \\
\text { lantana, mau-me- } \\
\text { quer }\end{array}$ & & & $\begin{array}{l}\text { purificação do sangue, } \\
\text { ajuda no parto, } \\
\text { rouquidão expectorante, } \\
\text { diurético, gripe, dor de } \\
\text { cabeça }\end{array}$ & $\begin{array}{l}\text { Caruaru (PE) } \\
\text { Cantareira (SP) } \\
\text { Cocal (PI) } \\
\text { Extrema (MG) } \\
\text { Fernão (MG) } \\
\text { Floresta (PE) } \\
\text { Florianópolis (SC) } \\
\text { Igarassu (PE) } \\
\text { Ilhéus (BA) } \\
\text { Ingaí (MG) } \\
\text { Itacaré (BA) } \\
\text { Itumirim (MG) } \\
\text { Itutinga (MG) } \\
\text { Lavras (MG) } \\
\text { Lima Duarte (MG) } \\
\text { Mato Grosso } \\
\text { Nova Friburgo (RJ) } \\
\text { Nova Iguaçu (RJ) } \\
\text { Paulista (PE) } \\
\text { Petrópolis (RJ) } \\
\text { Quissamã (RJ) } \\
\text { Rio de Janeiro (RJ) } \\
\text { Santo Antonio do } \\
\text { Leverger (MT) }\end{array}$ & $\begin{array}{l}\text { Chaves \& Barros (2012) } \\
\text { Costa \& Mayworm } \\
\text { (2011) } \\
\text { Costa et al. (2006) } \\
\text { Cunha \& Bortolotto } \\
\text { (2011) } \\
\text { Cunha et al. (2012) } \\
\text { Guarim Neto \& Morais } \\
\text { (2003) } \\
\text { Hoeffel et al. (2011) } \\
\text { Leitão et al. (2009) } \\
\text { Lorenzi \& Matos (2008) } \\
\text { Lucena et al. (2007) } \\
\text { Maioli-Azevedo \& } \\
\text { Fonseca-Kruel (2007) } \\
\text { Oliveira (2005) } \\
\text { Oliveira \& Menini Neto } \\
\text { (2012) } \\
\text { Pinto et al. (2006) } \\
\text { Rodrigues \& Carvalho } \\
\text { (2001) } \\
\text { Silva \& Andrade (2005) } \\
\text { Silva et al. (2010) } \\
\text { Sobrinho et al., (2011) }\end{array}$ \\
\hline $\begin{array}{l}\text { L. canescens } \\
\text { Kunth }\end{array}$ & Lantana & & Fo, FI & $\begin{array}{l}\text { Contusões, diurético, dor } \\
\text { de dente }\end{array}$ & Itapoá (SC) & $\begin{array}{l}\text { Merétika et al. (2010) } \\
\text { Silva (2012) }\end{array}$ \\
\hline $\begin{array}{l}\text { L. fucata Lindl. } \\
\text { [Sin.L. lilacina } \\
\text { Desf.] }\end{array}$ & $\begin{array}{l}\text { Cambará-roxo, } \\
\text { milho-cereja }\end{array}$ & & & $\begin{array}{l}\text { Inflamação de garganta, } \\
\text { tosse, gripe, bronquite } \\
\text { e asma }\end{array}$ & $\begin{array}{l}\text { Alto paraíso de } \\
\text { Goiás (GO) } \\
\text { Mato Grosso (MT) } \\
\text { Rio de Janeiro (RJ) }\end{array}$ & $\begin{array}{l}\text { Azevedo \& Silva (2006) } \\
\text { Guarim Neto \& Morais } \\
(2003) \\
\text { Silva (2012) } \\
\text { Souza \& Felfili (2006) }\end{array}$ \\
\hline $\begin{array}{l}\text { L. megapotamica } \\
\text { (Spreng.) Tronc. }\end{array}$ & Sabiá-do-mato & & & $\begin{array}{l}\text { Dores no estômago e } \\
\text { fígado }\end{array}$ & Ipê (RS) & Ritter et al. (2002) \\
\hline $\begin{array}{l}\text { Lantana radula } \\
\text { Sw. }\end{array}$ & & Decocção & Fo & Gripe, tosse, bronquite & Nordeste & Agra et al. (2008) \\
\hline L. trifolia $L$. & $\begin{array}{l}\text { Cidreira, uvinha- } \\
\text { do-campo }\end{array}$ & Chá & Fo & Antiúlcera & $\begin{array}{l}\text { Nossa Senhora do } \\
\text { Livramento (MT) }\end{array}$ & $\begin{array}{l}\text { Jesus et al. (2009) } \\
\text { Silva (2012) }\end{array}$ \\
\hline $\begin{array}{l}\text { L. undulata } \\
\text { Schrank }\end{array}$ & Camará-branco & & & & Ilhéus (BA) & $\begin{array}{l}\text { Costa et al. (2006) } \\
\text { Silva (2012) }\end{array}$ \\
\hline Lantana sp. & $\begin{array}{l}\text { Camará, } \\
\text { chumbinho, } \\
\text { moleque-duro }\end{array}$ & & Fo, FI & $\begin{array}{l}\text { Gripe, febre, asma, } \\
\text { tosse, bronquite, diabete, } \\
\text { pressão alta, calmante }\end{array}$ & $\begin{array}{l}\text { Delmiro Gouveia } \\
\text { (AL) } \\
\text { Piranhas (AL) }\end{array}$ & Almeida (2004) \\
\hline $\begin{array}{l}\text { Lippia alba (Mill.) } \\
\text { N.E.Br. }\end{array}$ & $\begin{array}{l}\text { Alecrim-do-campo, } \\
\text { alecrim-selvagem, } \\
\text { cambará- } \\
\text { de-espinho, } \\
\text { camelitana, } \\
\text { chá-de-tabuleiro, } \\
\text { cidreira, cidreira- } \\
\text { brava, cidreira- } \\
\text { carmelitana, } \\
\text { cidreira-de-folha- } \\
\text { redonda, cidrila, } \\
\text { erva-cidreira, } \\
\text { erva-cidreira-de- } \\
\text { arbusto, erva- } \\
\text { cidreira-do-campo, } \\
\text { erva-cidreira- } \\
\text { brasileira, falsa- } \\
\text { melissa, melissa }\end{array}$ & $\begin{array}{l}\text { Chá, } \\
\text { infusão, } \\
\text { banho e } \\
\text { decocção }\end{array}$ & $\begin{array}{l}\mathrm{Fo}, \mathrm{Cl}, \\
\mathrm{Sm}, \mathrm{FI}\end{array}$ & $\begin{array}{l}\text { Dor de barriga, "tudo", } \\
\text { barriga inchada, } \\
\text { comida que faz mal, } \\
\text { calmante, cólicas, gripe, } \\
\text { tosse, pressão alta, } \\
\text { cólica menstrual, dor } \\
\text { de estômago, cólica } \\
\text { intestinal, vômito, } \\
\text { diarreia, sedativo, febre, } \\
\text { desconforto, anemia, } \\
\text { insônia, rouquidão, dor } \\
\text { de cabeça, ameba, } \\
\text { gases, menopausa, } \\
\text { intestino, doenças } \\
\text { do sistema nervoso, } \\
\text { antidiarreico, } \\
\text { digestivo, anti- } \\
\text { espasmodico, menstrual, } \\
\text { depurativo, convulsão, } \\
\text { hipotensor }\end{array}$ & $\begin{array}{l}\text { Alagoinha (PE) } \\
\text { Amargosa (BA) } \\
\text { Anastácio (MS) } \\
\text { Apodi (RN) } \\
\text { Arraial do Cabo } \\
\text { (RJ) } \\
\text { Bandeirantes (PR) } \\
\text { Belém (PA) } \\
\text { Boa Vista (RR) } \\
\text { Buriti dos Montes } \\
\text { (PI) } \\
\text { Cananéia (SP) } \\
\text { Cantareira (SP) } \\
\text { Caruaru (PE) } \\
\text { Cascavel (PR) } \\
\text { Casimiro de Abreu } \\
\text { (RJ) } \\
\text { Caucaia (CE) } \\
\text { Crateús (CE) } \\
\text { Demerval Lobão } \\
\text { (PI) } \\
\text { Esperantina (PI) } \\
\text { Extrema (MG) }\end{array}$ & $\begin{array}{l}\text { Agra et al. (2008) } \\
\text { Aguiar \& Barros (2012) } \\
\text { Albertasse et al. (2010) } \\
\text { Albuquerque et al. } \\
\text { (2007) } \\
\text { Albuquerque (2006) } \\
\text { Albuquerque \& Andrade } \\
\text { (2002a) } \\
\text { Albuquerque \& Andrade } \\
\text { (2002b) } \\
\text { Alcântara Júnior et al. } \\
\text { (2005) } \\
\text { Almeida \& Albuquerque } \\
\text { (2002) } \\
\text { Amorozo (2002) } \\
\text { Araujo (2007) } \\
\text { Azevedo \& Silva (2006) } \\
\text { Brito \& Senna-Valle } \\
\text { (2011) } \\
\text { Christo et al. (2010) } \\
\text { Crepaldi (2007) } \\
\text { Costa \& Mayworm } \\
\text { (2011) } \\
\text { Cunha \& Bortolotto } \\
\text { (2011) }\end{array}$ \\
\hline
\end{tabular}


TABELA 1. Espécies medicinais da família Verbenaceae, seguidas pelo Nome Popular, Forma de Uso, Parte Usada, Indicações, Área de Estudo e Referências.

continuação...

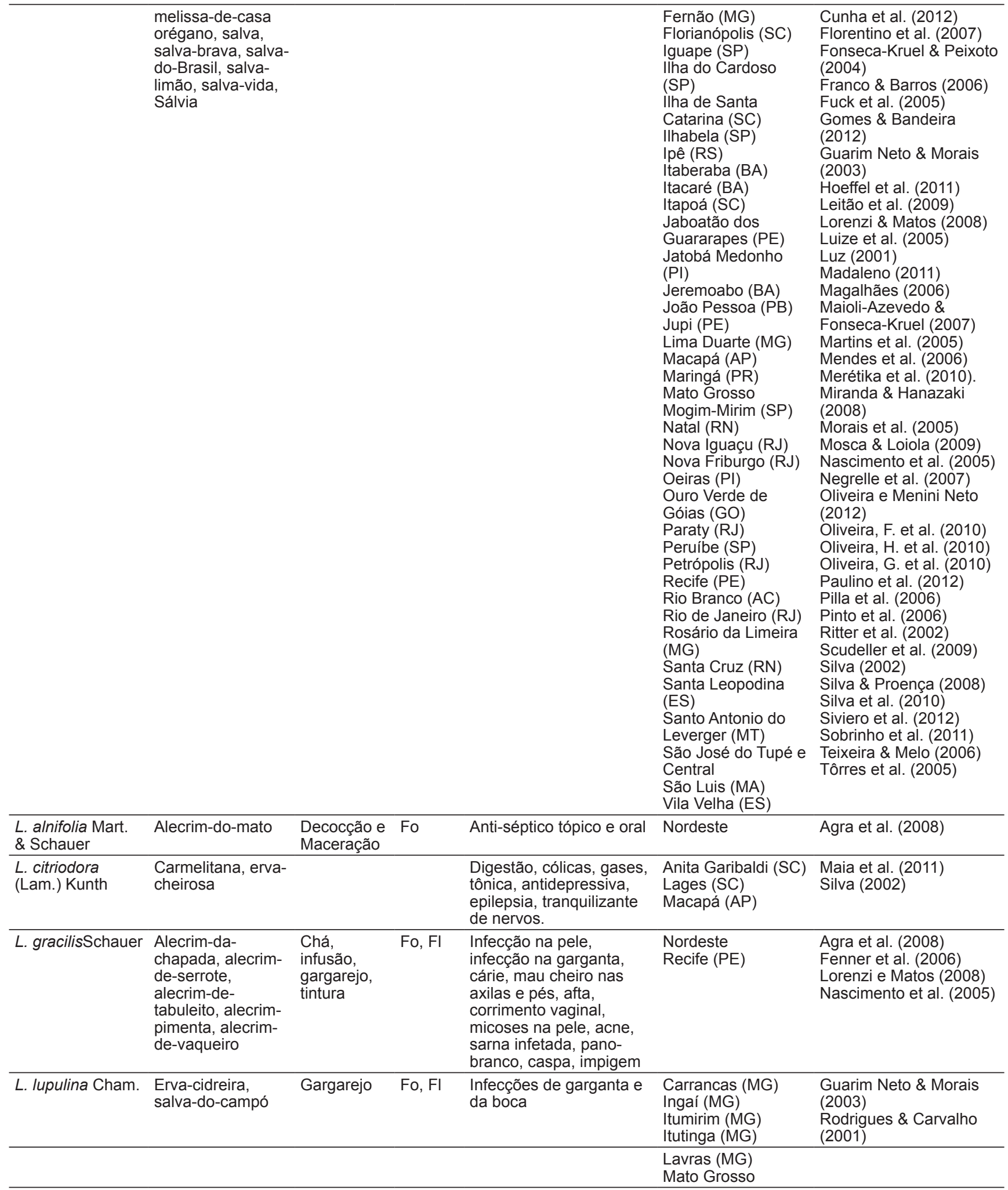

continua...

Rev. Bras. PI. Med., Campinas, v.17, n.4, supl. II, p.980-991, 2015. 
TABELA 1. Espécies medicinais da família Verbenaceae, seguidas pelo Nome Popular, Forma de Uso, Parte Usada, Indicações, Área de Estudo e Referências.

continuação...

\begin{tabular}{|c|c|c|c|c|c|c|}
\hline $\begin{array}{l}\text { Lippia microphylla } \\
\text { Cham. }\end{array}$ & $\begin{array}{l}\text { Salva-do-campo, } \\
\text { alecrim-da- } \\
\text { chapada, alecrim- } \\
\text { de-tabuleiro }\end{array}$ & $\begin{array}{l}\text { Inalação, } \\
\text { xarope }\end{array}$ & Fo & $\begin{array}{l}\text { Gripe, malária, } \\
\text { pneumonia, } \\
\text { antiflamatório, sinusite, } \\
\text { tosse, congestão nasal, } \\
\text { anti-séptico }\end{array}$ & $\begin{array}{l}\text { Amargosa (BA) } \\
\text { Boa Vista (RR) } \\
\text { Mutuípe (BA) } \\
\text { Nordeste } \\
\text { Recife (PE) }\end{array}$ & $\begin{array}{l}\text { Agra et al. (2008) } \\
\text { Lorenzi \& Matos (2008) } \\
\text { Luz (2001) } \\
\text { Nascimento et al. (2005) } \\
\text { Pinto \& Maduro (2003) } \\
\text { Silva et al. (2010) }\end{array}$ \\
\hline $\begin{array}{l}\text { L. origanoides } \\
\text { Kunth } \\
\text { [Sin.L. sidoides } \\
\text { Cham] }\end{array}$ & $\begin{array}{l}\text { Alecrim-bravo, } \\
\text { alecrim-do- } \\
\text { nordeste, alecrim- } \\
\text { pimenta, estrepa- } \\
\text { cavalo }\end{array}$ & Chá, Tintura & Fo, FI & $\begin{array}{l}\text { Anti-sépto local, caspa, } \\
\text { anti-fúgico local, } \\
\text { impingem, dermatoses, } \\
\text { rinite alérgica, infecção } \\
\text { na pele e garganta, } \\
\text { cárie, mal cheiro nas } \\
\text { axilas e pés, afta, } \\
\text { corrimento vaginal, } \\
\text { micoses. }\end{array}$ & $\begin{array}{l}\text { Custódia (PE) } \\
\text { Floresta (PE) } \\
\text { Recife (PE) }\end{array}$ & $\begin{array}{l}\text { Fenner et al. (2006) } \\
\text { Lorenzi \& Matos (2008) } \\
\text { Nascimento et al. (2005) }\end{array}$ \\
\hline $\begin{array}{l}\text { L. pseudothea } \\
\text { (A.St.-Hil.) } \\
\text { Schauer }\end{array}$ & Chá-de-pedestre & Xarope & Fo, FI & & & $\begin{array}{l}\text { Brandão et al. (2006) } \\
\text { Brandão et al. (2008) }\end{array}$ \\
\hline $\begin{array}{l}\text { L. thymoides } \\
\text { Mart. \& Schaurer }\end{array}$ & Alecrim & Chá; Banho & Fo & $\begin{array}{l}\text { Gripe, tosse, cansaço, } \\
\text { nariz entupido, febre, } \\
\text { reumatismo }\end{array}$ & $\begin{array}{l}\text { Amargosa (BA) } \\
\text { Boa vista (RR) } \\
\text { Jeremoabo (BA) }\end{array}$ & $\begin{array}{l}\text { Gomes \& Bandeira } \\
\text { (2012) } \\
\text { Silva et al. (2010) }\end{array}$ \\
\hline Lippia sp1. & Melissa & & & & $\begin{array}{l}\text { Amargosa (BA) } \\
\text { Boa vista (RR) } \\
\text { Mutuípe (BA) }\end{array}$ & Silva et al. (2010) \\
\hline \multirow[t]{2}{*}{ Lippia sp2. } & Alecrim-de- & & Fo, FI & & $\begin{array}{l}\text { Delmiro Gouveia } \\
(\mathrm{AL})\end{array}$ & Almeida (2004) \\
\hline & $\begin{array}{l}\text { vaqueiro, alecrim- } \\
\text { de-cabloco, } \\
\text { alecrim-pimenta }\end{array}$ & & & $\begin{array}{l}\text { Gripe, bronquite, } \\
\text { inflamação nos pulmões, } \\
\text { pneumonia, tirar catarro, } \\
\text { inflamação na garganta, } \\
\text { constipação, coração. }\end{array}$ & Piranhas $(A L)$ & \\
\hline Lippia sp3. & Alecrim & Decocção & Fo & $\begin{array}{l}\text { Gripe, anti-microbiano, } \\
\text { anti-sépco, digestão }\end{array}$ & $\begin{array}{l}\text { Buriti dos Montes } \\
\text { (PI) } \\
\text { Crateús (CE) }\end{array}$ & Magalhães (2006) \\
\hline Lippia sp4. & Erva-cidreira & & & & $\begin{array}{l}\text { Igarassu (PE) } \\
\text { Paulista (PE) }\end{array}$ & Silva \& Andrade (2005) \\
\hline Lippia sp5. & Alecrim & & & & Alagoinha (PE) & $\begin{array}{l}\text { Albuquerque \& Andrade } \\
(2002 b)\end{array}$ \\
\hline Lippia sp6. & Alecrim & Chá & Fo & $\begin{array}{l}\text { Dor de barriga, má } \\
\text { digestão. }\end{array}$ & Alagoinha (PE) & $\begin{array}{l}\text { Albuquerque \& Andrade } \\
(2002 a)\end{array}$ \\
\hline Lippia sp7. & Camarazinha & & & & $\begin{array}{l}\text { Alagoinha (PE) } \\
\text { Caruaru (PE) } \\
\text { Floresta (PE) } \\
\end{array}$ & Lucena et al. (2007) \\
\hline Lippia sp8. & Alecrim & Infusão & Fo & Digestão, Disenteria & Alagoinha (PE) & Albuquerque (2006) \\
\hline Lippia sp9. & Alecrim & $\begin{array}{l}\text { Decocção, } \\
\text { banho }\end{array}$ & Fo & Gripe & Oeiras (PI) & Oliveira, F. et al. (2010) \\
\hline Lippia sp10. & $\begin{array}{l}\text { Alecrim-de- } \\
\text { passos, alecrim- } \\
\text { de-bom-Jesus }\end{array}$ & Banho & Fo, FI & Sinusite & Oeiras (PI) & Oliveira, F. et al. (2010) \\
\hline Lippia sp11. & Erva-cidreira & Chá & Fo & & Lagarto (SE) & Silva et al. (2006) \\
\hline Lippia sp12. & Melissa & & & & Itacaré (BA) & Pinto et al. (2006) \\
\hline Petrea sp. & Espinheira-santa & & & Estômago & Mogim-Mirim (SP) & Pilla et al. (2006) \\
\hline $\begin{array}{l}\text { Stachytarpheta } \\
\text { angustifolia (Mill.) } \\
\text { Vahl } \\
\text { [Sin. } \\
\text { Stachytarpheta } \\
\text { elatior Schrad. ex } \\
\text { Schult.] }\end{array}$ & Gervão, mocotó & $\begin{array}{l}\text { Suco ou } \\
\text { sumo }\end{array}$ & Fo & & $\begin{array}{l}\text { Igarassu (PE) } \\
\text { Mato Grosso } \\
\text { Nova Xavantina } \\
\text { (MT) } \\
\text { Nordeste } \\
\text { Paulista (PE) }\end{array}$ & $\begin{array}{l}\text { Agra et al. (2008) } \\
\text { Guarim Neto \& Morais } \\
(2003) \\
\text { Silva \& Andrade (2005) }\end{array}$ \\
\hline
\end{tabular}


TABELA 1. Espécies medicinais da família Verbenaceae, seguidas pelo Nome Popular, Forma de Uso, Parte Usada, Indicações, Área de Estudo e Referências.

continuação...

\begin{tabular}{|c|c|c|c|c|c|}
\hline $\begin{array}{l}\text { S. cayennensis } \\
\text { (Rich.) Vahl } \\
\text { [Sin.S. dichotoma } \\
\text { (Ruiz \& Pav.) } \\
\text { Vahl] }\end{array}$ & $\begin{array}{l}\text { Aguarapondá, } \\
\text { aguare-ponda, } \\
\text { chá-de-burro, chá- } \\
\text { do-Brasil, erva-de- } \\
\text { grilo, erva-gervão, } \\
\text { ervão, falsa- } \\
\text { verbena, gervão, } \\
\text { gervão-azul, } \\
\text { gervão-do-campo, } \\
\text { gervão-legítimo, } \\
\text { gervão-preto, } \\
\text { gervão-roxo, } \\
\text { gervão-verdadeiro, } \\
\text { jarbão, mocotó, } \\
\text { orgibão, rincão, } \\
\text { rinchão, uregão, } \\
\text { urgevão, verbena, } \\
\text { verbena-falsa }\end{array}$ & $\begin{array}{l}\text { Decocção, } \\
\text { Bochecho, } \\
\text { xarope, } \\
\text { infusão }\end{array}$ & $\begin{array}{l}\mathrm{Fo}, \mathrm{Cl}, \\
\mathrm{Pt}\end{array}$ & $\begin{array}{l}\text { Tônico estomacal, } \\
\text { estimulante } \\
\text { gastrointestinal, febre, } \\
\text { dispepsia, diurético, } \\
\text { emoliente, problemas } \\
\text { hepáticos crônicos, anti- } \\
\text { helmíntico, vermífugo, } \\
\text { úlceras, sórdidas, } \\
\text { cicatrizante, anemia, } \\
\text { colesterol, problemas } \\
\text { de fígado, dores } \\
\text { estomacais, câncer, } \\
\text { glicose, diabetes, } \\
\text { emagrecer, vitamina, } \\
\text { amarelão (hepatite), triza } \\
\text { (hepatite), dor de dente, } \\
\text { dor de barriga, doenças } \\
\text { no aparelho respiratório, } \\
\text { dores no estômago, anti- } \\
\text { espasmódico }\end{array}$ & $\begin{array}{l}\text { Anastácio (MS) } \\
\text { Anita Garibaldi (SC) } \\
\text { Arraial do Cabo } \\
\text { (RJ) } \\
\text { Auto Paraíso de } \\
\text { Goiás (GO) } \\
\text { Cantareira (SP) } \\
\text { Carrancas (MG) } \\
\text { Casimiro de Abreu } \\
\text { (RJ) } \\
\text { Extrema (MG) } \\
\text { Fernão (MG) } \\
\text { Florianópolis (SC) } \\
\text { Governador } \\
\text { Valadares (MG) } \\
\text { Iguape (SP) } \\
\text { Ilhabela (SP) } \\
\text { Ingaí (MG) } \\
\text { Itacaré (BA) } \\
\text { Itapoá (SC) } \\
\text { Itumirim (MG) } \\
\text { Itutinga (MG) } \\
\text { Lages (SC) } \\
\text { Lavras (MG) } \\
\text { Lima Duarte (MG) } \\
\text { Macapá (AP) } \\
\text { Madureira (RJ) }\end{array}$ \\
\hline
\end{tabular}

Agra et al. (2008)

Amorozo (2002)

Araujo (2007)

Azevedo \& Silva (2006)

Boscolo \& Valle (2008)

Brandão et al. (2006)

Brandão et al. (2008)

Brasileiro et al. (2006)

Brito \& Senna-Valle

(2011)

Christo et al. (2010)

Costa \& Mayworm

(2011)

Costa et al. (2006)

Crepaldi (2007)

Cunha \& Bortolotto

(2011)

Feijó et al. (2012)

Fonseca-Kruel \& Peixoto

(2004)

Gandolfo \& Hanazaki

(2011)

Giraldi \& Hanazaki

(2010)

Guarim Neto \& Morais

(2003)

Hoeffel et al. (2011)

Jesus et al. (2009)

Leitão et al. (2009)

Lorenzi \& Matos (2008)

\begin{tabular}{|c|c|c|c|c|c|c|}
\hline & & & & & $\begin{array}{l}\text { Mangaratiba (RJ) } \\
\text { Mato Grosso } \\
\text { Nossa Senhora do } \\
\text { Livramento (MT) } \\
\text { Paraty (RJ) } \\
\text { Pelotas (RS) } \\
\text { Peruíbe (SP) } \\
\text { Quissamã (RJ) } \\
\text { Rio Branco (AC) } \\
\text { Rio de Janeiro (RJ) } \\
\text { Santa Leopodina } \\
\text { (ES) } \\
\text { Santo Antonio do } \\
\text { Leverger (MT) } \\
\text { São joão do Tupé e } \\
\text { Central } \\
\text { São Luis (MA) }\end{array}$ & $\begin{array}{l}\text { Madaleno (2011) } \\
\text { Maia et al. (2011) } \\
\text { Maioli-Azevedo \& } \\
\text { Fonseca-Kruel (2007) } \\
\text { Medeiros et al. (2004) } \\
\text { Medeiros et al. (2005) } \\
\text { Merétika et al. (2010) } \\
\text { Oliveira \& Menini Neto } \\
\text { (2012) } \\
\text { Pinto et al. (2006) } \\
\text { Rodrigues \& Carvalho } \\
\text { (2001) } \\
\text { Scudeller et al. (2009) } \\
\text { Silva (2002) } \\
\text { Siviero et al. (2012) }\end{array}$ \\
\hline $\begin{array}{l}\text { S. jamaicensis (L) } \\
\text { Vahl }\end{array}$ & $\begin{array}{l}\text { Aguara-ponda, } \\
\text { chá-do-Brasil, } \\
\text { gerbão, gervão, } \\
\text { gervão-azul, } \\
\text { orgibão, urgevão }\end{array}$ & Infusão & Fo & $\begin{array}{l}\text { Diabetes, úlceras } \\
\text { sórdidas, antimalária, } \\
\text { fígado, estômago }\end{array}$ & $\begin{array}{l}\text { Rosário da Limeira } \\
\text { (MG) } \\
\text { Vila Velha (ES) }\end{array}$ & $\begin{array}{l}\text { Albertasse et al. (2010) } \\
\text { Fenner et al. (2006) } \\
\text { Oliveira (2008) } \\
\text { Oliveira, H. et al. (2010) }\end{array}$ \\
\hline $\begin{array}{l}\text { S. polyura } \\
\text { Schauer }\end{array}$ & Gervão & & & & Mato Grosso & $\begin{array}{l}\text { Guarim Neto \& Morais } \\
(2003)\end{array}$ \\
\hline $\begin{array}{l}\text { S. schottiana } \\
\text { Schauer }\end{array}$ & Gervão-da-praia & & $\mathrm{Rz}$ & $\begin{array}{l}\text { Vermes, problemas no } \\
\text { fígado }\end{array}$ & Quissamã (RJ) & Boscolo \& Valle (2008) \\
\hline $\begin{array}{l}\text { Stachytarpheta } \\
\text { sp1. }\end{array}$ & Gervão & & & $\begin{array}{l}\text { Tônica, febrífuga, } \\
\text { infecções, vermífuga, } \\
\text { reduz colesterol }\end{array}$ & $\begin{array}{l}\text { Alto Paraíso de } \\
\text { Goiás (GO) }\end{array}$ & Souza \& Felfili (2006) \\
\hline \multicolumn{3}{|c|}{$\begin{array}{l}\text { Stachytarphetantinua... } \\
\text { sp2. }\end{array}$} & $\mathrm{Pt}$ & $\begin{array}{l}\text { Retenção de urina, } \\
\text { inflamação renal }\end{array}$ & $\begin{array}{l}\text { Delmiro Gouveia } \\
(\mathrm{AL}) \\
\text { Piranhas }(\mathrm{AL})\end{array}$ & Almeida (2004) \\
\hline $\begin{array}{l}\text { Verbena } \\
\text { intermedia Gillies } \\
\text { \& Hook. ex Hook }\end{array}$ & $\begin{array}{l}\text { Gervão-da-folha- } \\
\text { branca, fel-de- } \\
\text { terra }\end{array}$ & & & $\begin{array}{l}\text { Pontada no fígado, abrir } \\
\text { o apetite }\end{array}$ & Ipê (RS) & Ritter et al. (2002) \\
\hline V. litoralis Kunth & & & Fo & $\begin{array}{l}\text { Problemas de estômago, } \\
\text { problemas de fígado, } \\
\text { dores de estômago, } \\
\text { colesterol, leucemia }\end{array}$ & $\begin{array}{l}\text { Igarassu (PE) } \\
\text { Itapoá (SC) }\end{array}$ & Merétika et al. (2010) \\
\hline
\end{tabular}

continua...

Rev. Bras. PI. Med., Campinas, v.17, n.4, supl. II, p.980-991, 2015. 
TABELA 1. Espécies medicinais da família Verbenaceae, seguidas pelo Nome Popular, Forma de Uso, Parte Usada, Indicações, Área de Estudo e Referências.

\begin{tabular}{lllllll}
\hline $\begin{array}{l}\text { V. montevidensis } \\
\text { Spreng. }\end{array}$ & Gervão & $\begin{array}{l}\text { Decocção, } \\
\text { bochecho }\end{array}$ & Fo & Fígado (dor intoxicação) & Ipê (RS) & Ritter et al. (2002) \\
\hline V. officinalis L. & Vervena & & Fo & Feridas & Fenner et al. (2006) \\
\hline Indeterminada 1 & Narapiró & & & & Itacaré (BA) & Pinto et al. (2006) \\
\hline Indeterminada 2 & Alfazema II & Itacaré (BA) & Pinto et al. (2006) \\
\hline Indeterminada 3 & $\begin{array}{l}\text { Erva-cidreira-de- } \\
\text { canelinha-rosa }\end{array}$ & Chá & Fo & Jupi (PE) & Teixeira \& Melo (2006) \\
\hline Indeterminada 4 & Hortelão & $\begin{array}{l}\text { Decocção, } \\
\text { lambedor, } \\
\text { inalação }\end{array}$ & Fo, Fl & Gripe & Oeiras (PI) & Oliveira, F. et al. (2010) \\
\hline
\end{tabular}

Nome científico: (Sin.: sinonímias). Parte usada: (Fo: Folha, FI: Flor, Fr: Fruto, Rz: Raiz, Cl: Caule, Sm: Semente; Pt: Planta toda). Área de Estudo: (SC: Santa Catarina, PR: Paraná, RS: Rio Grande do Sul; RJ: Rio de Janeiro, ES: Espírito Santo; AP: Amapá; GO: Goiás; MS: Mato Grosso do Sul, PE: Pernambuco, Bahia: Bahia, MG: Minas Gerais, RR: Roraima, SP: São Paulo, PI: Piauí, MT: Mato Grosso, AL: Alagoas, RN: Rio Grande do Norte, PA: Pará, CE: Ceará, AC: Acre, MA: Maranhão, SE: Sergipe).

mostram que a mesma possui propriedades medicinais em função da atividade sedativa de seu óleo essencial produzido de óleos pelos seus tricomas secretores.

Do levantamento das espécies medicinais realizado nos trabalhos que serviram como base para esta pesquisa, pode-se constatar que os trabalhos com maior riqueza de espécies medicinais da família Verbenaceae foram: Guarim Neto \& Morais (2003) e Agra et al. (2008) com oito registros cada, seguidos de Pinto et al. (2006) com seis, Merétika et al. (2010), Fenner et al. (2006) e Silva et al. (2010) com cinco espécies cada

Alguns autores listaram espécies que atualmente são consideradas sinônimas. Dessa forma, foram agrupadas todas as informações referentes às espécies sinônimas, dentre elas estão: Aloysia citriodora Palau [= A. triphylla (L'Hér.) Britton]; A. lycioides Cham. [= A. pulchra (Briq.) Moldenke]; Bouchea fluminensis (Vell.) Moldenke [= B. pseudogervao (A.St.-Hil.) Cham.]; Casselia chamaedryfolia Cham. [= C. mansoi Schauer]; Lantana fucata Lindl. [= L. lilacina Desf.]; Lippia origanoides Kunth [ $=$ L. sidoides Cham]; Stachytarpheta angustifolia (Mill.) Vahl $[=S$. elatior Schrad. ex Schult.] e S. cayennensis (Rich.) Vahl [= S. dichotoma (Ruiz \& Pav.) Vahl].

Não foram levadas em consideração as espécies que não pertencem atualmente ao grupo das Verbenaceae e que foram registrados nos trabalhos analisados como sendo desse grupo, como por exemplo, Vitex spp. (Lamiaceae).

Em relação à utilização das plantas descritas na literatura a parte mais utilizada foi a folha com 32 indicações, seguida pela flor (11). A forma de uso mais referenciada foi o chá (=infusão) com 16 citações. As patologias mais citadas foram referentes aos problemas respiratórios e digestivos.

Os dados deste trabalho corroboram com as pesquisas de Aguiar \& Barros (2012) e Albertasse et al. (2010) onde afirmaram que a folha e o chá também foram à parte usada e o modo de preparo respectivamente mais citados.

Estudos sobre diversidade e uso de recursos medicinais, realizados por Chaves \& Barros (2012), assim como, estudos sobre utilização de plantas medicinais por Costa \& Mayworm (2011), corroboraram com os dados levantados nesse trabalho sobre as categorias de indicações das plantas medicinais sendo que o maior número de indicações referiam-se ao tratamento das doenças do sistema respiratório e do aparelho digestório.

Quanto à área de estudo dos artigos analisados, todas as regiões foram contempladas, sendo que os Estados que apresentaram o maior número de trabalhos publicados foram Rio de Janeiro com 11 registros, seguidos de Minas Gerais e Pernambuco com sete trabalhos cada.

Amorozo (2002) afirmou que embora algumas populações encontrem facilidades médicas, como centros de saúde e hospitais, o uso de plantas com fins terapêuticos faz parte do cotidiano da maioria das pessoas.

Badke et al. (2012) e Borba \& Macedo (2006), afirmaram que o conhecimento sobre as plantas medicinais é adquirido ao longo do tempo, seja por meio de transmissão oral, ou do conhecimento iniciado na infância e passado de geração para geração.

Desse modo, o uso de plantas medicinais está cada vez mais presente entre as comunidades, fazendo com que a importância de estudos nesta área seja cada vez mais ampliada. Com isso, as informações contidas neste trabalho servirão para auxiliar na elaboração de banco de dados sobre o modo de uso das plantas pertencentes à família Verbenaceae com potencial medicamentoso encontradas nos Estados brasileiros. 


\section{REFERÊNCIAS}

AGRA, M.F. et al. Survey of medicinal plants used in the region Northeast of Brazil. Brazilian Journal of Pharmacognosy, v. 18, n. 3, p. 472-508, 2008.

AGUIAR, L.C.G.G.; BARROS, R. F. M. Plantas medicinais cultivadas em quintais de comunidades rurais no domínio do cerrado piauiense (Município de Demerval Lobão, Piauí, Brasil). Revista Brasileira de Plantas Medicinais, v. 14, n. 3, p. 419-434, 2012.

ALBERTASSE, P.D. et al. Plantas medicinais e seus usos na comunidade da Barra do Jucu, Vila Velha, ES. Revista Brasileira de Plantas Medicinais, v. 12, n. 3, p. 250-260, 2010.

ALBUQUERQUE, U.P.; ANDRADE, L.H.C. Conhecimento Botânico Tradicional e Conservação em uma Área de Caatinga no Estado de Pernambuco, Nordeste do Brasil. Acta Botanica Brasilica, v. 16, n. 3, p. 273-285, 2002a.

ALBUQUERQUE, U.P.; ANDRADE, L.H.C. Uso de Recursos Vegetais da Caatinga: o Caso do Agreste do Estado de Pernambuco (Nordeste do Brasil). Interciencia, v. 27, n. 7, p. 336-346, 2002b.

ALBUQUERQUE, U.P. et al. Use of plant resources in a seasonal dry forest (Northeastern Brazil). Acta Botanica Brasilica, v. 19, n. 1, p. 27-38, 2005.

ALBUQUERQUE, U.P. et al. Medicinal and magic plants from a public market in northeastern Brazil. Journal of Ethnopharmacology, v. 110, p. 76-91, 2007.

ALBUQUERQUE, U.P. Re-examining hypotheses concerning the use and knowledge of medicinal plants: a study in the Caatinga vegetation of NE Brazil. Journal of Ethnobiology and Ethnomedicine, v. 2, p. 1-30, 2006.

ALCÂNTARA JÚNIOR, J.P. et al. Levantamento Etnobotânico e Etnofarmacológico de Plantas Medicinais do Município de Itaberaba-BA Para Cultivo e Preservação. Sitientibus, v. 5, n. 1, p. 39-44, 2005.

ALMEIDA, C.F.C.B.R.; ALBUQUERQUE, U.P. Uso e Conservação de Plantas e Animais medicinais no estado de Pernambuco (Nordeste do Brasil): Um estudo de caso. Juvencia, v. 27, n. 6, p. 276-285, 2002.

ALMEIDA, C.F.C.B.R. Etnobotânica nordestina: Estratégia de vida e composição química como preditores do uso de plantas medicinais por comunidades locais na caatinga. 2004. $66 \mathrm{f}$. Dissertação (Mestrado - Área de Concentração em Etnobotânica) - Departamento de Botânica, Universidade Federal de Pernambuco, Departamento de Botânica, Recife.

ALVES, T. M.A. et al. Biological Screening of Brazilian Medicinal Plants. Memórias do Instituto Oswaldo Cruz, v. 95, n. 3, p. 367-373, 2000.

AMOROZO, M.C.M. Uso e Diversidade de Plantas Medicinais em Santo Antonio do Leverger, MT, Brasil. Acta botânica brasílica, v. 16, n. 2, p. 189-203, 2002.

APG III. An update of the Angiosperm Phylogeny Group classifi cation for the orders and families of fl owering plants: APG III. Botanical Journal of the Linnean Society, v. 161, p. 105-121, 2009.

ARAÚJO, L.G. Etnobotânica Caiçara: diversidade e conhecimento de recursos vegetais no litoral paulista. 2007. 195f. Dissertação (Mestrado - Área de Concentração em Ecologia) - Instituto de Biologia, Universidade Estadual de Campinas, Campinas.
AZEVEDO, S.K.S.; SILVA, I.M. Plantas medicinais e de uso religioso comercializadas em mercado e feiras livres no Rio de Janeiro, RJ, Brasil. Acta Botanica Brasilica, v. 20, n. 1, p. 185-194, 2006.

BADKE, M.R. et al. Saberes e Práticas Populares de Cuidado em Saúde com o Uso de Plantas Medicinais. Texto \& Contexto Enfermagem, v. 21, n. 2, p. 363370, 2012.

BORBA, A.M.; MACEDO, M. Plantas medicinais usadas para a saúde bucal pela comunidade do bairro Santa Cruz dos Guimarães, MT, Brasil. Acta Botanica Brasilica, v. 20, n. 4, p. 771-782, 2006.

BORGES, R.; PEIXOTO, A.L. Conhecimento e uso de plantas em uma comunidade caiçara do litoral sul do Estado do Rio de Janeiro, Brasil. Acta Botanica Brasilica, v. 23, n. 3, p. 769-779, 2009.

BOSCOLO, O.H.; VALLE, L.S. Plantas de uso medicinal em Quissamã, Rio de Janeiro, Brasil. Iheringia, v. 63, n. 2, p. 263-277, 2008.

BOTREL, R.T. et al. Uso da vegetação nativa pela população local no município de Ingaí, MG, Brasil. Acta Botanica Brasilica, v. 20, n. 1, p. 143-156, 2006.

BRANDÃO, M.G.L. et al. Medicinal plants and other botanical products from the Brazilian Official Pharmacopoeia. Brazilian Journal of Pharmacognosy, v. 16, n. 3, p. 408-420, 2006.

BRANDÃO, M.G.L. et al. Other medicinal plants and botanical products from the first edition of the Brazilian Official Pharmacopoeia. Brazilian Journal of Pharmacognosy, v. 18, n. 1, p. 127-134, 2008.

BRASILEIRO, B.G. et al. Antimicrobial and cytotoxic activities screening of some Brazilian medicinal plants used in Governador Valadares district. Brazilian Journal of Pharmaceutical Sciences, v.42, n. 2, p. 195-202, 2006.

BRITO, M.R.; SENNA-VALLE, L. Plantas medicinais utilizadas na comunidade caiçara da Praia do Sono, Paraty, Rio de Janeiro, Brasil. Acta Botanica Brasilica, v. 25, n. 2, p. 363-372, 2011.

BUENO, O.L.; LEONHARDT, C. Distribuição e potencial paisagístico dos gêneros Citharexylum L. e Verbenoxylum Tronc. no Rio Grande do Sul, Brasil. Iheringia, v. 66, n. 1, p. 47-60, 2011.

CAMÊLO, L.C.A. et al. Caracterização morfológica e agronômica de acessos de erva-cidreira-brasileira [Lippia alba (Mill.) N. E. Br.]. Scientia Plena, v. 7, n. 5, p. 1-8, 2011.

CHAVES, E.M.F.; BARROS, R.F.M. Diversidade e uso de recursos medicinais do carrasco na APA da Serra da Ibiapaba, Piauí, Nordeste do Brasil. Revista Brasileira de Plantas Medicinais, v. 14, n. 3, p. 476-486, 2012.

CHRISTO, A.G. et al. Local knowledge on medicinal plant gardens in a rural community near the Atlantic Rain Forest, southeastern Brazil. Brazilian Journal of Pharmacognosy, v. 20, n. 4, p. 494-501, 2010.

COSTA, L.C.B. et al. Levantamento preliminar das espécies vegetais com potencial econômico no parque municipal da Boa Esperança, Ilhéus, Bahia, Brasil. Acta Farmacêutica Bonaerense, v. 25, n. 2, p. 184-191, 2006.

COSTA, V.P.; MAYWORM, M.A.S. Plantas medicinais utilizadas pela comunidade do bairro dos Tenentes, município de Extrema, MG, Brasil. Revista Brasileira

Rev. Bras. PI. Med., Campinas, v.17, n.4, supl. II, p.980-991, 2015. 
de Plantas Medicinais, v. 13, n. 3, p. 282-292, 2011. CREPALDI, M.O.S. Etnobotânica na Comunidade Quilombola Cachoeira do Retiro, Santa Leopoldina, Espírito Santo, Brasil. 2007. 65f. Dissertação (Mestrado - Área de Concentração em Botânica) - Instituto de Pesquisas Jardim Botânico do Rio de Janeiro, Escola Nacional de Botânica Tropical, Rio de Janeiro.

CUNHA LIMA, S.T. et al. The use of medicinal plants by an indigenous Pataxó community in NE Brazil. Revista Brasileira de Plantas Medicinais, v. 14, n. 1, p. 8491, 2012.

CUNHA, S.A.; BORTOLOTTO, I.M. Etnobotânica de Plantas Medicinais no Assentamento Monjolinho, município de Anastácio, Mato Grosso do Sul, Brasil. Acta Botanica Brasilica, v. 25, n. 3, p. 685-698, 2011.

FAVORITO, S. Tricomas Secretores de Lippia stachyoides Cham. (Verbenaceae): Estrutura, Ontogênese e Secreção. 2009. 89f. Dissertação (Mestrado - Área de Concentração em Morfologia e Diversidade Vegetal) - Instituto de Biociências, Universidade Estadual Paulista (UNESP), Botucatu.

FEIJÓ, A.M. et al. Plantas medicinais utilizadas por idosos com diagnóstico de Diabetes mellitus no tratamento dos sintomas da doença. Revista Brasileira de Plantas Medicinais, v. 14, n. 1, p. 50-56, 2012.

FENNER, R. et al. Plantas utilizadas na medicina popular brasileira com potencial atividade antifúngica. Revista Brasileira de Ciências Farmacêuticas, v. 42, n. 3, p. 369-394, 2006.

FLORENTINO, A.T.N. et al. Contribuição de quintais agroflorestais na conservação de plantas da Caatinga, Município de Caruaru, PE, Brasil. Acta Botânica Brasileira, v. 21, n. 1, p. 37-47, 2007.

FONSECA-KRUEL, V.S.; PEIXOTO, A.L. Etnobotânica na Reserva Extrativista Marinha de Arraial do Cabo, RJ, Brasil. Acta Botanica Brasilica, v. 18, n. 1, p. 177-190, 2004.

FRANCO, E.A.P.; BARROS, R.F.M. Uso e diversidade de plantas medicinais no Quilombo Olho D'água dos Pires, Esperantina, Piauí. Revista Brasileira de Plantas Medicinais, v. 8, n. 3, p. 78-88, 2006.

FUCK, S.B. et al. Plantas medicinais utilizadas na medicina popular por moradores da área urbana de Bandeirantes, PR, Brasil. Semina, v. 26, n. 3, p. 291296, 2005.

GANDOLFO, E.S.; HANAZAKI, N. Etnobotânica e urbanização: conhecimento e utilização de plantas de restinga pela comunidade nativa do distrito do Campeche (Florianópolis, SC). Acta Botanica Brasilica, v. 25, n. 1, p. 168-177, 2011.

GIRALDI, M.; HANAZAKI, N. Uso e conhecimento tradicional de plantas medicinais no Sertão do Ribeirão, Florianópolis, SC, Brasil. Acta Botanica Brasilica, v. 24, n. 2, p. 395-406, 2010.

GOMES, T.B.; BANDEIRA, F.P.S.F. Uso e diversidade de plantas medicinais em uma comunidade quilombola no Raso da Catarina, Bahia. Acta Botanica Brasilica, v. 26, n. 4, p. 796-809, 2012.

GUARIM NETO, G.; MORAIS, R.G. Recursos Medicinais de Espécies do Cerrado de Mato Grosso: Um estudo bibliográfico. Acta Botanica Brasilica, v. 17, n. 4, p. 561-584, 2003.
HOEFFEL, J.L.M. et al. Conhecimento Tradicional e Uso de Plantas Medicinais nas APA'S Cantareira/SP e Fernão Dias/MG. Revista VITAS, v. 1, p. 1-25, 2011.

JESUS, N.Z.T. et al. Levantamento etnobotânico de plantas popularmente utilizadas como antiúlceras e antiinflamatórias pela comunidade de Pirizal, Nossa Senhora do Livramento-MT, Brasil. Revista Brasileira de Farmacognosia, v. 19, n. 1, p. 130-139, 2009.

$\mathrm{KOCH}$, I. et al. Apocynaceae in Lista de Espécies da Flora do Brasil. Jardim Botânico do Rio de Janeiro. Disponível em: <http://floradobrasil.jbrj.gov.br/jabot/ floradobrasil/FB48>. Acesso em: 10 Jul. 2014.

LEITÃO, F. et al. Urban ethnobotany in Petrópolis and Nova Friburgo (Rio de Janeiro, Brazil). Brazilian Journal of Pharmacognosy, v. 19, n. 1, p. 333-342, 2009.

LORENZI, H.; MATOS, F.J.A. Plantas medicinais no Brasil: Nativas e Exóticas. Nova Odessa (SP): Instituto Plantarum, $2^{\mathrm{a}}$ ed., 2008. 544 p.

LUCENA, R.F.P. et al. Useful Plants of the SemiArid Northeastern Region Of Brazil: A Look at their Conservation and Sustainable Use. Environ Monit Assess, v. 125, p. 281-290, 2007.

LUIZE, P.S. et al. Effects of medicinal plant extracts on growth of Leishmania (L.) amazonensis and Trypanosoma cruzi. Brazilian Journal of Pharmaceutical Sciences, v. 41, n. 1, p. 85-94, 2005

LUZ, F.J.F. Plantas medicinais de uso popular em Boa Vista, Roraima, Brasil. Horticultura Brasileira, v. 19, n. 1, p. 88-96, 2001.

MACIEL, M.A.M. et al. Plantas Medicinais: a necessidade de estudos multidisciplinares. Química Nova, v. 25, n. 3, p. 429-438, 2002.

MADALENO, I.M. Plantas da medicina popular de São Luís, Brasil. Boletim do Museu Paraense Emílio Goeldi, v. 6, n. 2, p. 273-286, 2011.

MAGALHÃES, A. Perfil Etnobotânico e Conservacionista das Comunidades do Entorno da Reserva Natural Serra das Almas, Ceará-Piauí, Brasil. 2006. 68f. Dissertação (Mestrado - Área de Concentração em Desenvolvimento e Meio Ambiente) - PRODEMA, Universidade Federal do Ceará, Fortaleza.

MAIA, E.A. et al. O uso de espécies vegetais para fins medicinais por duas comunidades de Serra Catarinense, Santa Catarina, Brasil. Revista de Biologia e Ciências da Terra, v. 11, n. 1, p. 54-74, 2011.

MAIOLI-AZEVEDO, V.; FONSECA-KRUEL, V.S. Plantas medicinais e ritualísticas vendidas em feiras livres no Município do Rio de Janeiro, RJ, Brasil: estudo de caso nas zonas Norte e Sul. Acta Botanica Brasilica, v. 21, n. 2, p. 263-275, 2007.

MARTINS, A.G. et al. Levantamento etnobotânico de plantas medicinais, alimentares e tóxicas da Ilha do Combu, Município de Belém, Estado do Pará, Brasil. Revista Brasileira de Farmácia, v. 86, n. 1, p. 21-30, 2005.

MEDEIROS, M.F.T. et al. Plantas medicinais e seus usos pelos sitiantes da Reserva Rio das Pedras, Mangaratiba, RJ, Brasil. Acta Botanica Brasilica, v. 18, n. 2, p. 391399, 2004.

MEDEIROS, M.F.T. et al. Flora Medicinal dos Sitiantes da Reserva Particular do Patrimônio Natural Rio das Pedras, Mangaratiba, Rio de Janeiro, Brasil: Taxonomia

Rev. Bras. PI. Med., Campinas, v.17, n.4, supl. II, p.980-991, 2015. 
e Aspectos Etnobotânicos. Publicações avulsas do Museu Nacional, v.106, p.3-24, 2005.

MELO, J.I.M. et al. Verbenaceae Sensu lato em um trecho da Esec Raso da Catarina, Bahia, Brasil. Revista Caatinga, v. 23, n. 3, p. 41-47, 2010.

MENDES, B.G. et al. Triagem de glicolipídios em plantas medicinais. Revista Brasileira de Farmacognosia, v. 16, n. 4, p. 568-575, 2006.

MERÉTIKA, A.H.C. et al. Local knowledge of medicinal plants in three artisanal fi shing communities (Itapoá, Southern Brazil), according to gender, age, and urbanization. Acta Botanica Brasilica, v. 24, n. 2, p. 386-394, 2010.

MING, L.C. et al. Espécies brasileiras com potencial alimentar: uso atual e perspectivas. In: CONGRESSO NACIONAL DE BOTÂNICA, 51, 2000, Brasília. Palestras Tópicos atuais em botânica. Brasília, DF: SBB/Embrapa-Cenargen, 2000. p. 268-273

MIRANDA, T.M.; HANAZAKI, N. Conhecimento e uso de recursos vegetais de restinga por comunidades das ilhas do Cardoso (SP) e de Santa Catarina (SC), Brasil. Acta Botanica Brasilica, v. 22, n. 1, p. 203-215, 2008.

MORAIS, S.M. et al. Plantas medicinais usadas pelos índios Tapebas do Ceará. Revista Brasileira de Farmacognosia, v. 15, n. 2, p. 169-177, 2005.

MOSCA, V.P.; LOIOLA, M.I.B. Uso Popular de Plantas Medicinais no Rio Grande do Norte, Nordeste do Brasil. Revista Caatinga, v. 22, n. 4, p. 225-234, 2009.

NASCIMENTO, J.E. et al. Produtos à base de Plantas Medicinais comercializados em Pernambuco - Nordeste do Brasil. Acta Farmacêutica Bonaerense, v. 24, n. 1, p. 113-22, 2005.

NEGRELLE, R.R.B. et al. Estudo etnobotânico junto à Unidade Saúde da Família Nossa Senhora dos Navegantes: subsídios para o estabelecimento de programa de fitoterápicos na Rede Básica de Saúde do Município de Cascavel (Paraná). Revista Brasileira de Plantas Medicinais, v. 9, n. 3, p. 6-22, 2007.

OLIVEIRA, E.R.; MENINI NETO, L. Levantamento etnobotânico de plantas medicinais utilizadas pelos moradores do povoado de Manejo, Lima Duarte - MG. Revista Brasileira de Plantas Medicinais, v. 14, n. 2, p. 311-320, 2012.

OLIVEIRA, F.C.S. et al. Plantas medicinais utilizadas em comunidades rurais de Oeiras, semiárido piauiense. Revista Brasileira de Plantas Medicinais, v. 12, n. 3, p. 282-301, 2010.

OLIVEIRA, G.L. et al. Plantas medicinais utilizadas na comunidade urbana de Muribeca, Nordeste do Brasil. Acta Botanica Brasilica, v. 24, n. 2, p. 571-577, 2010. OLIVEIRA, H.B. Estudo Etnofarmacológico de Plantas Medicinais em Rosário da Limeira-MG. 2008. 84f. Dissertação (Mestrado - Área de Concentração em Magister Scientiae) - Departamento de Fitotecnia, Universidade Federal de Viçosa, Viçosa.

OLIVEIRA, H.B. et al. Ethnopharmacological study of medicinal plants used in Rosário da Limeira, Minas Gerais, Brazil. Brazilian Journal of Pharmacognosy, v. 20, n. 2, p. 256-260, 2010.

OLIVEIRA, R.L.C. Prioridades de Conservação e Sustentabilidade do Extrativismo de Plantas Medicinais da Caatinga. 2005. 75f. Dissertação (Mestrado - Área de Concentração em Botânica) -
Departamento de Biologia, Universidade Federal Rural de Pernambuco (UFRPE), Recife.

PARENTE, C.E.T.; ROSA, M.M.T. Plantas comercializadas como medicinais no Município de Barra do Piraí, RJ. Rodriguésia, v. 52, n. 80, p. 47-59, 2001.

PAULINO, R.C. et al. Medicinal plants at the Sítio do Góis, Apodi, Rio Grande do Norte State, Brazil. Brazilian Journal of Pharmacognosy, v.22, n.1, p. 29-39, 2012.

PILLA, M.A.C. et al. Obtenção e uso das plantas medicinais no distrito de Martim Francisco, Município de Mogi-Mirim, SP, Brasil. Acta Botanica Brasilica, v. 20, n. 4, p. 789-802, 2006.

PINTO, E.P.P. et al. Conhecimento popular sobre plantas medicinais em comunidades rurais de mata atlântica Itacaré, BA, Brasil. Acta Botanica Brasilica, v. 20, n. 4, p. 751-762, 2006.

RITTER, M.R. et al. Plantas usadas como medicinais no município de Ipê, RS, Brasil. Revista Brasileira de Farmacognosia, v. 12, n. 2, p. 51-62, 2002.

RODRIGUES, V.E.G.; CARVALHO, D.A. Levantamento Etnobotânico de Plantas Medicinais no Domínio do Cerrado na região do Alto Rio Grande-Minas Gerais. Ciência e Agrotecnologia, v. 25, n. 1, p. 102-123, 2001.

SALIMENA, F.R.G. et al. Verbenaceae in Lista de Espécies da Flora do Brasil. Disponível em : <http:// floradobrasil.jbrj.gov.br/jabot/floradobrasil/FB246> Acesso em: 01 Abr. 2013.

SANTOS, F.M. et al. Produção de mudas de Aloysia gratissima (Gillies \& Hook.) Tronc. por meio da propagação sexuada e assexuada. Revista Brasileira de Plantas Medicinais, v. 11, n. 2, p. 130-136, 2009.

SCUDELLER, V.V. et al. Etnoconhecimento de plantas de uso medicinal nas comunidades São João do Tupé e Central (Reserva de Desenvolvimento Sustentável do Tupé). In: SANTOS-SILVA, E.N.; SCUDELLER, V.V. (Orgs.). Biotupé: Meio Físico, Diversidade Biológica e sociocultural do Baixo Rio Negro, Amazônia Central. Manaus: UEA Edições, 2009, p. 185-199.

SILVA, A.J.R.; ANDRADE, L.H.C. Etnobotânica nordestina: estudo comparativo da relação entre comunidades e vegetação na Zona do Litoral - Mata do Estado de Pernambuco, Brasil. Acta Botanica Brasilica, v. 19, n. 1, p. 45-60, 2005.

SILVA, C.S.P.; PROENÇA, C.E.B. Uso e disponibilidade de recursos medicinais no município de Ouro Verde de Goiás, GO, Brasil. Acta Botanica Brasilica, v. 22, n. 2, p. 481-492, 2008.

SILVA, M.P.L. et al. Levantamento Etnobotânico e Etnofarmacológico de Plantas Medicinais em Comunidades Rurais de Amargosa e Mutuípe - BA. Magistra, v. 22, n. 1, p. 8 -13, 2010.

SILVA, R. B. L. A Etnobotânica de Plantas Medicinais da Comunidade Quilombola de Curiaú, MacapáAP, Brasil. 2002. 172f. Dissertação (Mestrado - Área de Concentração em Agronomia) - Departamento de Agronomia, Universidade Federal Rural da Amazônia, Belém.

SILVA, T.R.S. Lantana in Lista de Espécies da Flora do Brasil. Disponível em: <http://floradobrasil.jbrj.gov. br/2012/FB015169> Acesso em: 11 Dez. 2012.

SIVIERO, A. et al. Plantas medicinais em quintais urbanos de Rio Branco, Acre, Revista Brasileira de Plantas Medicinais, v. 14, n. 4, p. 598-610, 2012.

Rev. Bras. PI. Med., Campinas, v.17, n.4, supl. II, p.980-991, 2015. 
SOBRINHO, F.A.P. et al. Uso de plantas medicinais no entorno da Reserva Biológica de Tinguá, Nova Iguaçu, RJ. Revista Acadêmica de Ciências Agrária e Ambiental, v. 9, n. 2, p. 195-206, 2011.

SOUZA, C.D.; FELFILI, J.M. Uso de plantas medicinais na região de Alto Paraíso de Goiás, GO, Brasil. Acta Botanica Brasilica v. 20, n. 1, p. 135-142, 2006.

TEIXEIRA, S.A.; MELO, J.I.M. Plantas medicinais utilizadas no município de Jupi, Pernambuco, Brasil. Iheringia, v. 61, n. 1-2, p. 5-11, 2006.

TROPICOS. Apocynaceae Juss. Disponível em: <http:// www.tropicos.org/> Acesso em: 10 Jul. 2014.
TÔRRES, A.R. et al. Estudo sobre o uso de plantas medicinais em crianças hospitalizadas da cidade de João Pessoa: riscos e benefícios. Revista Brasileira de Farmacognosia, v. 15, n.4, p. 373-380, 2005.

VANDRESEN, F. Constituição química, atividades antibacteriana, antiedematogênica e toxicidade frente à Artemia salina da espécie vegetal Aloysia gratissima (Gillies \& Hook.) Troncoso (Verbenaceae). 2005. 166f. Dissertação (Mestrado - Área de Concentração em Química) - Centro de Ciências Exatas, Universidade Estadual de Maringá, Maringá. 